DOSSIER

\title{
INVESTIGACIONES ARQUEOMALACOLÓGICAS EN SUDAMÉRICA: ENTRE LA SUBSISTENCIA, LO UTILITARIO Y LO SIMBÓLICO
}

\section{ARQUEOMALACOLOGICAL RESEARCH IN SOUTH AMERICA: BETWEEN SUBSISTENCE, UTILITY AND SYMBOLISM}

\author{
Sandra Gordilloํ, Andrés Gascue ${ }^{2}$ y Sebastián Pastor ${ }^{3}$ \\ ${ }^{1}$ Facultad de Ciencias Físicas, Exactas y Naturales, Universidad Nacional de Córdoba. Centro de \\ investigaciones en Ciencias de la Tierra. CONICET. Av. Vélez Sársfield 1611, X5016CGA, \\ Ciudad Universitaria, Córdoba, Argentina. sandra.gordillo@unc.edu.ar \\ ${ }^{2}$ Universidad de la República Oriental del Uruguay, Centro Universitario Regional del Este, \\ Rutas 9 y 15. Rocha, Uruguay. andresgascue@gmail.com \\ ${ }^{3}$ Centro de Investigación y Transferencia de Catamarca (CITCA-CONICET). S. Fernando del \\ Valle de Catamarca, Argentina. pastorvcp@yahoo.com.ar
}

La malacología en contextos arqueológicos tuvo, en los últimos años y a nivel mundial, un notable impulso con un aumento considerable del número de publicaciones en este campo multi e interdisciplinar donde convergen (a través de los moluscos como eje organizador) las ciencias naturales y sociales.

En Latinoamérica se destaca como uno de los antecedentes el Simposio Arqueología y Malacología: casos de estudio, perspectivas de análisis y abordajes metodológicos, realizado en el marco del Primer Congreso Argentino de Malacología (2013) y que contó con la participación de profesionales de la arqueología y la biología.

La idea de realizar el presente volumen surgió luego de la realización del Primer Simposio Latinoamericano de Arqueomalacología, en el marco del $\mathrm{X}$ Congreso Latinoamericano de Malacología (X CLAMA) que tuvo lugar el día 2 de octubre de 2017 en el Argentino Hotel de la ciudad de Piriápolis.

Respecto a la relación del Homo sapiens con los moluscos, actualmente se conoce que la especie humana ya consumía moluscos hace unos 300.000 años (Classeen 1998). No obstante, además de la dieta, muchos grupos humanos de la antigüedad utilizaron también sus conchas con diversos propósitos. En tal sentido, entre los hallazgos que tendrían mayor antigüedad podemos mencionar al sitio Skhul, en Asia occidental (con edades entre 100.000 y 135.000 años; Vanhaeren et al. 2006) y la Cueva de Blombos, en Sudáfrica (aproximadamente 76.000 años; Henshilwood et al. 2004) en que se recuperaron cuentas realizadas con la concha de caracoles marinos del género Nassarius (respectivamente, N. gibbosulus y N. kraussianus). A partir de allí hay registros de su utilización como elementos utilitarios tales como monedas e instrumentos; y también en ámbitos simbólicos como medio de transmisión de información social (ornamentos, ofrendas funerarias, etc.). 
A nivel latinoamericano existen trabajos centrados en las acumulaciones de conchas a lo largo de las costas tanto del Atlántico como del Pacífico (ej. sambaquís en Brasil, concheros o conchales en Argentina, Chile y Uruguay), y otros que destacan a los artefactos de concha en cuanto a su uso tecnológico, valoración simbólica y, los menos, en la participación en redes de intercambio inter-regionales, como el caso tan emblemático del Spondylus.

Sin embargo, el desarrollo de la arqueomalacología en Latinoamérica es aún incipiente, quedando un inmenso territorio del conocimiento con vastas áreas aún sin explorar. Por ejemplo, la utilización de otros moluscos (además de Spondylus) para evaluar redes de intercambio o la reconstrucción de los paleoambientes y climas donde vivieron los antiguos grupos humanos. Por ello resulta necesario el desarrollo de mayores vínculos entre investigadores de las ciencias sociales y naturales en torno a este eje organizador, para la consolidación de nuevos abordajes inter, multi y transdisciplinarios que contribuyan al crecimiento sostenible de este campo de investigación.

El Simposio mencionado brindó una gran oportunidad para el intercambio de ideas y experiencias entre diferentes grupos de investigadores, principalmente de Argentina y Uruguay. Para este dossier se contó además con la contribución de colegas de Brasil, quedando reflejado, a través de los 10 trabajos que conforman este volumen, un panorama actualizado con amplia información sobre los avances y enfoques del abordaje de los problemas de índole arqueomalacológico en los países mencionados.

El artículo de Ortiz y colaboradores, titulado Arqueomalacología en el sitio arqueológico Pozo de la Chola (Región del Piedemonte de Jujuy, Argentina). Estudios tafonómicos, inferencias ambientales y procesos antrópicos analiza los restos de moluscos gasterópodos que aparecen con frecuencia en sitios del noroeste de Argentina, asignados al período Formativo temprano (500 AC-600 DC). Los distintos indicadores brindaron información sobre los agentes tafonómicos y antrópicos involucrados en su distribución, permitiendo además reconocer la prevalencia de la familia Ampullariidae, que tiende a concentrarse en sectores específicos vinculados con áreas de procesamiento.

En tanto la propuesta de Tissera y colaboradores, Entre Borus y fragmentos. Análisis de un contexto arqueomalacológico de producción de cuentas en Cerro Colorado (Sierras del Norte, Córdoba, Argentina) realiza el análisis de un conjunto de fragmentos malacológicos pertenecientes al caracol gigante Megalobulimus lorentzianus (Doering) procedente de una excavación efectuada en la localidad de Cerro Colorado (provincia de Córdoba). Los resultados obtenidos sugieren origen antrópico, y se interpreta a los sitios de procedencia de estos hallazgos, como un locus de producción que registra diferentes momentos de una cadena operativa, y se estima una cronología compatible con el período Prehispánico tardío propuesto para las Sierras Centrales (ca. 1500-400 $\mathrm{AP})$. 
El trabajo de Berón, Artefactos malacológicos. Diferentes contextos de uso en sitios arqueológicos de la Provincia de La Pampa (Argentina), aborda los materiales malacológicos procedentes tanto de sitios residenciales como mortuorios y se reconoce su importancia económica, social y simbólica. La autora destaca la relación de estos artefactos con las redes de movilidad e interacción de los cazadores-recolectores pampeanos.

Por su parte, Cuentas y tembetás malacológicos de los grupos cazadores-recolectores prehispánios del humedal del Paraná Inferior de Buc, Acosta y Loponte analiza ambos tipos de artefactos dado que constituyen el conjunto más numeroso de adornos para el Holoceno tardío de esta región. Los autores plantean su funcionalidad como demarcadores sociales en base a su distribución espacial y patrones morfológicos y morfométricos.

En el trabajo de Gascue y colaboradores denominado El rol de los moluscos en las poblaciones prehispánicas de Uruguay los autores presentan una síntesis de los datos referentes al manejo diferencial de materiales malacológicos realizado por las poblaciones cazadoras-recolectoras prehispánicas que ocuparon las regiones Este, Centro-Sur y Oeste de Uruguay durante el Holoceno tardío, con especial referencia a la identificación taxonómica y a los usos inferidos. Los autores concluyen que en estos contextos los moluscos se asocian principalmente a aspectos simbólicos (ornamentales y funerarios) de estas culturas, estando también representadas actividades económicas vinculadas al consumo alimentario y producción de artefactos utilitarios.

En el artículo Los moluscos fluviales en la subsistencia de los grupos cazadoresrecolectores del humedal del Paraná inferior de Loponte y colaboradores, se discute el rol de los moluscos fluviales en la subsistencia de los grupos cazadores-recolectores de esta región. En esta propuesta los autores analizan la disponibilidad ambiental del bivalvo Diplodon (Rhipidodonta) variabilis como parte de un proceso regional de diversificación e intensificación de la subsistencia durante el Holoceno tardío, analizando además las señales isotópicas de su ingesta.

Además, en el trabajo de Prous y Pessoa, titulado O registro de moluscos nos vestígios arqueológicos pré-históricos do Brasil los autores resaltan la importancia que los moluscos tuvieron en períodos prehistóricos en esta vasta región. Dentro de los diversos usos, destacan los moluscos litoraleños en la alimentación y el aprovechamiento de sus conchas como material constructivo en los sambaquis costeros, como instrumentos (para cortar, raspar, aserrar, perforar y aplanar), como recipientes, como adornos (collares, pulseras, adornos labiales) para indumentaria y como antiplástico en las cerámicas amazónicas. También se discute el significado de la presencia de conchas de gasterópodos del litoral encontradas en sitios interiores, dada la presencia de elementos prehistóricos y elementos intrusivos que podrían llevar a interpretaciones erróneas por parte de los arqueólogos.

En el artículo Estudios arqueomalacológicos en concheros de la costa norte de Santa Cruz, Patagonia Argentina: Alcances y Resultados Hammond presenta los aportes que resultaron de prospecciones intensivas complementadas con excavaciones estratigráficas que se corresponden al Holoceno tardío, lo que ha permitido 
caracterizar el material, teniendo en cuenta aspectos taxonómicos, paleoambientales, tafonómicos y de índole tecnológico, brindado información sobre diversas características del pasado humano relacionadas con la subsistencia, las conductas de recolección y los modos de ocupación del espacio.

Por otro lado, Zubimendi y colaboradores, en el trabajo titulado Identificación de cuentas de collar de Margarella (Thiele, 1893) en un entierro prehistórico en la desembocadura del río Santa Cruz (Patagonia Argentina), estudian y contextualizan en el ámbito regional de la Patagonia continental argentina y el archipiélago Fueguino, los restos arqueomalacológicos correspondientes, a un entierro individual, parcialmente excavado y datado en ca. 1100 años AP.

Finalmente, en La elaboración de cuentas con valvas de moluscos en Patagonia a través de la arqueología experimental, Leonardt presenta los resultados de un programa experimental desarrollado para analizar el patrón de producción y distribución de este tipo de artefactos en Patagonia durante el Holoceno tardío, a fin de evaluar su papel como medios de comunicación visual a escala macro-regional.

Así, y en base a estos 10 casos de estudio e investigación arqueomalacológica, en Sudamérica, acontecidos en diferentes contextos arqueológicos, es posible reconocer la utilización de diferentes especies de moluscos marinos, fluviales y terrestres, según la disponibilidad en las diferentes regiones; el uso de moluscos fluviales y marinos como medio de subsistencia; la presencia de moluscos en el ámbito doméstico y funerario; la existencia de prácticas cotidianas de fabricación y especialización dentro de una cadena de producción y distribución de artefactos, y la vinculación con redes de intercambio; y el valor simbólico de determinadas especies o como elementos de comunicación social. Todo esto permite concluir la importancia económica, social y simbólica de los moluscos en diferentes espacios y temporalidades de Sudamérica.

Queremos expresar un profundo agradecimiento a los 20 investigadores que participaron del proceso de evaluación y que con sus críticas, comentarios y aportes desde España, Francia, México, Brasil, Chile, Uruguay y Argentina enriquecieron la versión final de los trabajos: Esteban Álvarez-Fernández, Bárbara Avezuela Aristu, Víctor Bejega García, Laura Beovide, Mariano Bonomo, Caroline Borges, Jesús Cantillo, José Angel Expósito Álvarez, Carola Flores, Gala García-Argudo Ripoll, Luis GómezGastelum, Igor Gutiérrez-Zugasti, Emiliano Melgar, Jordi Nadal Lorenzo, Ximena Power, Francisco Pugliese, Fernando Santiago, Catalina Soto Rodríguez, Ester Verdún y Miquel Angel Vicens. Gracias!

Un agradecimiento especial al equipo editorial de Comechingonia, particularmente a Andrea Recalde y Sebastián Pastor, por todo el apoyo, acompañamiento y esfuerzo brindado para poder concretar este volumen. 


\section{Bobliografía citada}

Classeen, Ch.

1998 Shells Cambridge Manuals in Archaeology Series. xiv266 pp. Cambridge, New York, Melbourne: Cambridge University Press.

Henshilwood, C. S., D'Errico, F., Vanhaeren, M., Van Niekerk, K. y Jacobs, Z. 2004 Middle Stone Age shell beads from South Africa. Science, 304: 404.

Vanhaeren M., d'Errico, F., Stringer C., James, S.L., Todd, J.A. y Mienis H.K.

2006 Middle Paleolithic Shell Beads in Israel and Algeria. Science 312: 1785-1788 